\title{
Anti-angiogenesis boosts chemo-immunotherapy in patients with EGFR mutations or baseline liver metastases: insights from IMpower150 study
}

\author{
Sara Elena Rebuzzi ${ }^{1,2}$, Francesco Facchinetti $^{3}$, Marcello Tiseo $^{1,4}$ \\ ${ }^{1}$ Medical Oncology Unit, University Hospital of Parma, Parma, Italy; ${ }^{2}$ Medical Oncology Unit 1, Ospedale Policlinico San Martino, University of \\ Genova, Genova, Italy; ${ }^{3}$ INSERM U981, Gustave Roussy Cancer Campus, Université Paris Saclay, Villejuif, France; ${ }^{4}$ Department of Medicine and \\ Surgery, University of Parma, Parma, Italy \\ Correspondence to: Francesco Facchinetti, MD MSc. INSERM U981, Gustave Roussy Cancer Campus, 114 Rue Edouard Vaillant, 94800 Villejuif, \\ France. Email: francescofacchinetti2@gmail.com. \\ Comment on: Reck M, Mok TSK, Nishio M, et al. Atezolizumab plus bevacizumab and chemotherapy in non-small-cell lung cancer (IMpower150): \\ key subgroup analyses of patients with EGFR mutations or baseline liver metastases in a randomised, open-label phase 3 trial. Lancet Respir Med \\ 2019;7:387-401.
}

Submitted May 25, 2019. Accepted for publication Jun 12, 2019.

doi: $10.21037 /$ tcr.2019.06.21

View this article at: http://dx.doi.org/10.21037/tcr.2019.06.21

\section{Introduction}

The first-line treatment scenario of advanced non-small cell lung cancer (NSCLC) has been substantially revolutionized in the very last years, since immunotherapy has become a cornerstone.

In patients whose tumors lack driver oncogenes (i.e., $E G F R$ mutations or $A L K$ rearrangements) and express PD-L1 on at least $50 \%$ of tumor cells, single-agent pembrolizumab has been approved for its superior efficacy over standard platinum doublet-chemotherapy (1). In order to improve survival in NSCLC patients beyond the PD-L1 highly expressing subgroup, clinical trials assessing immune checkpoint inhibitor (ICI) combination therapies have been led, proving them as suitable new treatment strategies. Combinations of anti-PD-1/anti-PD-L1 antibodies with either standard chemotherapy or ipilimumab (anti-CTLA-4) have shown positive results and are defined as current or upcoming standards of care (2-8).

In addition, IMpower150 trial has explored the addition of the angiogenic inhibitor bevacizumab to the combination of the anti-PD-L1 agent atezolizumab and chemotherapy $(9,10)$. Besides known anti-angiogenic properties, antiVEGF agents have immunomodulatory effects, with reversal of VEGR-mediated immunosuppressive tumor microenvironment, which could enhance the anti-tumor activity of immunotherapy $(11,12)$. The addition of bevacizumab to immunotherapy and chemotherapy turned out to be a potential treatment strategy in defined NSCLC subgroups, namely for EGFR-mutated tumors and for cases with baseline liver metastases (10). Both disease subgroups are of clear clinical interest, given the limited activity and efficacy of immunotherapy in the pretreated setting (13-15), making the results in first-line combinations a relevant step for the beneficial incorporation of ICIs in their management.

Albeit this commentary is specifically directed to the subgroup analyses of IMpower150 recently published (10), a global view of the trial is provided. Retracing the relative segmented evidence is not immediate given the complex design of the study, involving three treatment arms, whose results have been reported separately, and the presence of several co-primary, secondary and exploratory endpoints of clinical interest.

\section{IMPOWER150—primary analyses}

IMpower150 is the first phase III trial evaluating the combination of chemotherapy (carboplatin and paclitaxel) with atezolizumab and bevacizumab in the first-line therapy of advanced non-squamous NSCLC, including oncogeneaddicted tumors. Patients with EGFR or $A L K$ alterations were included after disease progression or intolerance to at 
Table 1 Main results of the IMpower 150 trial

\begin{tabular}{|c|c|c|c|c|c|c|c|c|c|c|c|c|}
\hline Treatment & $\begin{array}{c}\text { Pts } \\
\text { ABCP, } \\
\mathrm{N}[\%]\end{array}$ & $\begin{array}{c}\text { Pts } \\
\text { BCP, } \\
\mathrm{N}[\%]\end{array}$ & $\begin{array}{l}\text { mPFS, } \\
\text { ABCP } \\
\text { (months) }\end{array}$ & $\begin{array}{c}\text { mPFS, } \\
\text { BCP } \\
\text { (months) }\end{array}$ & $\mathrm{HR}(95 \% \mathrm{Cl})$ & $\begin{array}{c}\text { mOS } \\
\text { ABCP } \\
\text { (months) }\end{array}$ & $\begin{array}{r}\text { mOS BCP } \\
\text { (months) }\end{array}$ & $\mathrm{HR}(95 \% \mathrm{Cl})$ & $\begin{array}{c}\text { ORR } \\
\text { ABCP } \\
(\%)\end{array}$ & $\begin{array}{c}\text { ORR } \\
\text { BCP } \\
(\%)\end{array}$ & $\begin{array}{c}\text { mDOR } \\
\text { ABCP } \\
\text { (months) }\end{array}$ & $\begin{array}{c}\text { mDOR } \\
\text { BCP } \\
\text { (months) }\end{array}$ \\
\hline $\begin{array}{l}\text { WT } \\
\text { population }\end{array}$ & 356 [89] & 336 [84] & 8.3 & 6.8 & $\begin{array}{c}0.62 \\
(0.52-0.74)\end{array}$ & 19.2 & 14.7 & $\begin{array}{c}0.78 \\
(0.64-0.96)\end{array}$ & 63.5 & 48 & 9 & 5.7 \\
\hline
\end{tabular}

WT, wild type; Pts, patients; N, number; ABCP, atezolizumab plus bevacizumab plus carboplatin and paclitaxel; BCP, bevacizumab plus carboplatin and paclitaxel; mPFS, median progression-free survival; HR, hazard ratio; $\mathrm{Cl}$, confidence interval; mOS, median overall survival; ORR, overall response rate; mDOR, median duration of response; WT, wild-type; Teff-high WT, WT population with high T-effector gene-signature expression; NA, not available.

least one approved tyrosine kinase inhibitor (TKI) (9).

A total of 1,202 patients were randomised to receive atezolizumab plus bevacizumab plus carboplatin and paclitaxel (ABCP) (400 patients), atezolizumab plus carboplatin and paclitaxel (ACP) (402 patients) and bevacizumab plus carboplatin and paclitaxel (BCP) (400 patients). The primary analysis evaluated the efficacy of ABCP compared to BCP (9). Stratification factors for randomization included the presence of liver metastases, while only patients with treated brain lesions could be enrolled. Of note, the study was amended to exclude oncogene-addicted cases from the primary-analysis population, moving from the intention-to-treat (ITT) to the wild-type (WT) populations, due to the limited role of ICIs in EGFR-mutated and ALK-rearranged NSCLC. In addition, owing to the relevancy of immune-related gene expression profiles addressing the benefit of atezolizumab (16), the WT population with high expression of effector T-cell gene signature (Teff-high WT population) substituted the PD-L1-positive one in defining the other group of interest for primary endpoints analyses.

Socinski and collaborators indeed shown, as co-primary endpoints, a significant improvement in progression-free survival (PFS) with ABCP compared to BCP in the WT population [8.3 vs. 6.8 months; hazard ratio (HR) 0.62; $95 \%$ confidence interval $(\mathrm{CI}), 0.52-0.74 ; \mathrm{P}<0.001]$ and in the Teff-high WT population (11.3 vs. 6.8 months; HR $0.51 ; 95 \%$ CI, 0.38-0.68; $\mathrm{P}<0.001)$. Still in the context of co-primary endpoints, a longer overall survival (OS) in the WT population was observed for ABCP compared to BCP [median OS (mOS) 19.2 vs. 14.7 months; HR 0.78; 95\% CI, 0.64-0.96; $\mathrm{P}=0.02$ ] (Table 1).

As secondary endpoints, ABCP was associated with longer PFS in the ITT population and in all exploratory subgroups, which include EGFR- and ALK-positive patients, the Teff-low WT population, all PD-L1 expression subgroups, patients with liver metastases and those with KRAS mutation (9).

\section{IMPOWER150_key subgroup analyses}

Reck and collaborators (10) published thereafter the results of the pre-specified key secondary and exploratory endpoints in all treatment arms (ABCP, BCP and ACP) and in-patient subgroups which included EGFR-positive patients, patients with baseline liver metastases and with differential PD-L1 expression, as well as the ITT population data.

Among the 124 EGFR-positive patients ( $10 \%$ of the ITT population), 91 (73\%) harbored a "sensitising" EGFR mutation (exon 19 deletion and L858M mutations). Among this latter group, 13 patients (14\%) had not received any approved TKI before randomization. As the presence of EGFR mutations was not a stratification factor, 34 patients were allocated into the $\mathrm{ABCP}$ arm, whereas 45 each in the BCP and ACP ones. Of note, only 10 patients comprehensively had previously received osimertinib.

EGFR-mutated patients exposed to ABCP experienced longer OS compared to BCP-treated ones: after a median follow-up of 19.6 months, mOS were indeed respectively not estimated (NE; 95\% CI, 17-NE months) and 18.7 months (95\% CI, 13.4-NE months), leading to an OS HR of 0.61 (95\% CI, 0.29-1.28). Of note, the crossing of the upper bound of $95 \%$ CI over 1 was attributed to the limited number of patients included in the analysis, affecting its statistical power (10). Longer median PFS (mPFS; 10.2 vs. 6.9 months; HR 0.61; 95\% CI, 0.36-1.03) 
Table 2 Results of the treatment subgroups of IMpower 150

\begin{tabular}{|c|c|c|c|c|c|c|c|}
\hline Treatment & EGFR+ & Sensitising EGFR+ & Liver metastases & PD-L1 negative & PD-L1 low & PD-L1 high & ITT \\
\hline Pts ABCP, N [\%] & $34[9]$ & $24[6]$ & $52[13]$ & $190[48]$ & 135 [34] & 75 [19] & 400 \\
\hline Pts BCP, N [\%] & $45[11]$ & $32[8]$ & $57[14]$ & $200[50]$ & $127[32]$ & $73[18]$ & 400 \\
\hline mPFS ABCP (months) & 10.200 & 10.300 & 8.200 & NA & NA & NA & 8.400 \\
\hline $\mathrm{HR}(95 \% \mathrm{Cl})$ & $\begin{array}{c}0.61 \\
(0.36-1.03)\end{array}$ & $\begin{array}{c}0.41 \\
(0.23-0.75)\end{array}$ & $\begin{array}{c}0.41 \\
(0.26-0.62)\end{array}$ & $\begin{array}{c}0.75 \\
(0.60-0.94)\end{array}$ & $\begin{array}{c}0.55 \\
(0.42-0.73)\end{array}$ & $\begin{array}{c}0.33 \\
(0.22-0.51)\end{array}$ & $\begin{array}{c}0.59 \\
(0.50-0.69)\end{array}$ \\
\hline mOS ABCP (months) & NE & NE & 13.3 & 17.1 & 22.5 & 25.2 & 19.8 \\
\hline mOS BCP (months) & 18.7 & 17.5 & 9.4 & 14.4 & 16.7 & 13.2 & 14.9 \\
\hline $\mathrm{HR}(95 \% \mathrm{Cl})$ & $\begin{array}{c}0.61 \\
(0.29-1.28)\end{array}$ & $\begin{array}{c}0.31 \\
(0.11-0.83)\end{array}$ & $\begin{array}{c}0.52 \\
(0.33-0.82)\end{array}$ & $\begin{array}{c}0.83 \\
(0.64-1.08)\end{array}$ & $\begin{array}{c}0.76 \\
(0.54-1.08)\end{array}$ & $\begin{array}{c}0.67 \\
(0.42-1.06)\end{array}$ & $\begin{array}{c}0.76 \\
(0.63-0.93)\end{array}$ \\
\hline ORR BCP (\%) & 42 & NA & 41 & 37 & 41 & 49 & 40 \\
\hline mDOR ABCP (months) & 11.1 & 11.1 & 10.7 & NA & NA & NA & 11.5 \\
\hline mDOR BCP (months) & 4.7 & NA & 4.6 & NA & NA & NA & 6 \\
\hline \multicolumn{8}{|l|}{ ACP vs. BCP } \\
\hline Pts ACP, N [\%] & $45[11]$ & $33[8]$ & $53[13]$ & $185[46]$ & $148[37]$ & $68[17]$ & 402 \\
\hline Pts BCP, N [\%] & $45[11]$ & $32[8]$ & $57[14]$ & $200[50]$ & 127 [32] & $73[18]$ & 400 \\
\hline mPFS ACP (months) & 6.900 & 6.000 & 5.400 & 5.500 & 6.900 & NA & NA \\
\hline mPFS BCP (months) & 6.900 & 6.100 & 5.400 & 6.900 & 6.200 & NA & NA \\
\hline ORR ACP (\%) & 36 & NA & 27 & NA & NA & 62 & 41 \\
\hline ORR BCP (\%) & 42 & NA & 41 & NA & NA & 49 & 40 \\
\hline mDOR ACP (months) & 5.6 & NA & 5.6 & NA & NA & NA & 8.3 \\
\hline mDOR BCP (months) & 4.7 & NA & 4.6 & NA & NA & NA & 6 \\
\hline
\end{tabular}

ITT, intention-to-treat; TKI, tyrosine kinase inhibitor; ABCP, atezolizumab plus bevacizumab plus carboplatin and paclitaxel; BCP, bevacizumab plus carboplatin and paclitaxel; Pts, patients; N, number; mPFS, median progression-free survival; NA, not available; HR, hazard ratio; $\mathrm{Cl}$, confidence interval; mOS, median overall survival; ORR, overall response rate; mDOR, median duration of response; ACP, atezolizumab plus carboplatin and paclitaxel.

and duration of response [duration of response (DOR) 11.1 vs. 4.7 months] were observed in the ABCP compared to the $\mathrm{BCP}$ arms, as well as a higher overall response rate(ORR; 71\% vs. $42 \%)$. The best outcomes in terms of ABCP were obtained in patients with "sensitising" EGFR mutations (Table 2). No better survival and response benefits were observed in EGFRmutated patients treated with ACP compared to BCP (Table 2).

Patients with liver metastases at baseline $(\sim 13 \%)$ experienced longer OS (13.3 vs. 9.4 months; HR 0.52; 95\% CI, 0.33-0.82), PFS (8.2 vs. 5.4 months; HR 0.41; 
95\% CI, 0.26-0.62), mDOR (median DOR; 10.7 vs. 4.6 months) and higher ORR (61\% vs. $41 \%$ ) with ABCP compared to BCP. No survival improvement and a lower response rate were observed in liver metastases patients treated with ACP compared to BCP (Table 2).

In the ITT population, patients were divided according to PD-L1 expression in both tumor and immune cells (TC and IC, respectively) in PD-L1-negative (TC0 and IC0), PD-L1low (TC1/2 or IC1/2: PD-L1 expression on $\geq 1 \%$ and $<50 \%$ of tumor cells and $\geq 1 \%$ and $<10 \%$ of tumor-infiltrating immune cells) and PD-L1-high (TC3 or IC3) patients (9). The ABCP regimen improved OS, PFS, $\mathrm{mDOR}$ and ORR compared to BCP across all PD-L1 expression subgroups, especially in PDL1-high patients (Table 2). The ACP treatment was associated with higher PFS and ORR compared to BCP only in PD-L1high patients, while mDOR was longer with ACP compared to $\mathrm{BCP}$ across all PD-L1 subgroups. In the ITT population, ABCP shown improved OS (19.8 vs. 14.9 months; HR 0.76; 95\% CI, 0.63-0.93), PFS (8.4 vs. 6.8 months; HR 0.59; 95\% CI, $0.50-0.69$ ), and ORR (56\% vs. $40 \%$ ) compared to BCP. A survival benefit in terms of OS was observed, even though less significant, also with ACP compared to BCP (mOS 19.5 vs. 14.9 months; HR 0.85; 95\% CI, 0.71-1.03) (Table 2).

\section{Discussion}

IMpower 150 is the first randomised phase III trial of the combination of immunotherapy, chemotherapy and antiangiogenic therapy, allowing oncogene-addicted NSCLC to be included.

The study shown a survival advantage with the addition of atezolizumab to BCP as first-line therapy in advanced non-squamous NSCLC. This benefit was observed not only in the WT population but also in previously treated EGFRmutated NSCLC, patients with baseline liver metastases and with low/negative PD-L1 expression, subgroups where ICI monotherapy shown limited effect in previous studies.

Concerning oncogene-addicted diseases, whereas huge improvements in ALK-driven disease survival have been observed thanks to the availability of several generations of TKI, prolonged disease control in EGFR-positive ones appears more challenging, mainly relying on osimertinib administration in first- or second line $(17,18)$. Therefore, there is a critical need for this epidemiologically relevant group of patients, who are not suitable candidate to ICI monotherapy in later lines of treatment (13). The combination treatment evaluated in IMpower150 brings hope in this sense, envisaging the incorporation of immunotherapy even in EGFR-positive cases, potentially thanks to the synergistic effect provided by the angiogenic blockade.

The rationale of this trial and the explanation of the survival benefit in these clinically relevant subgroups have strong biological bases, which is one of the main strengths of this study. The hypothesis of this study was based not only on the synergistic effects of combining immunotherapy with chemotherapy-induced neoantigen release but also on the immunomodulatory effects of antiVEGF therapy. High intratumoral VEGF levels suppress the maturation of dendritic cells, promote the proliferation of regulatory $T$ cells and induce the expansion of immature myeloid cells. They lead also to abnormal growth of tumor vessels that induced a hypoperfused and hypoxic tumor microenvironment which impedes the T-effector cell infiltration contributing to tumor immune evasion $(11,19)$. Tumor cells can adapt to a hypoxic microenvironment by expressing hypoxic-inducible factor $1 \alpha$ (HIF1A) protein, which promotes the expression of PD-L1. The correlation between PD-L1 and hypoxia-related genes (such as HIF1A and $V E G F$ ) has been identified especially in hepatocellular carcinoma (HCC) but also in NSCLC (20). Liver metastases of NSCLC are associated with few T-effector cells infiltration which is the basis of a status of liverinduced immunotolerance, associated with a decreased likelihood of response to immunotherapy (21).

Moreover, VEGF and EGFR signaling have many overlapping and parallel downstream pathways: EGFR activation has been shown to promote $V E G F$ expression and blocking VEGF signaling result in downregulation of EGFR signaling (22). Therefore, anti-angiogenic agents induce an immune reprogramming of the tumor microenvironment from an immune-suppressive to an immune-permissive one.

The additive benefit of bevacizumab to the combination of immunotherapy and chemotherapy observed in these key patient subgroups is further supported by the absence of survival benefit of the combination ACP compared to BCP. Moreover, the survival benefit reported with $\mathrm{ABCP}$ in EGFR-positive patients and patients with liver metastases did not appear to be driven by high PD-L1 expression.

In EGFR-mutated NSCLC the beneficial addition of bevacizumab is also supported by the recent results of another trial on immunotherapy which included EGFR/ ALK-positive patients. The IMpower130 (8), unlikely IMpower 150, did not show a survival benefit of the immunotherapy/chemotherapy combination compared to chemotherapy alone in this patient subgroup, in contrast 
to the ITT WT population (23). Of note, US Food and Drug Administration (FDA) approved atezolizumab in combination with bevacizumab, carboplatin and paclitaxel in untreated advanced non-squamous NSCLC lacking EGFR or $A L K$ molecular alterations, whereas European Medicines Agency (EMA) approval recommendation included patients with EGFR-mutant or ALK-positive NSCLC after failure of appropriate targeted therapies.

One of the major limitations of this study is the small sample size of the reported subgroups, with special regard to the EGFR-positive population (Table 2), and the exploratory nature (even though pre-specified) of the patient subgroup analyses. As mentioned above, it is relevant that oncogeneaddicted NSCLC were firstly excluded from the primary endpoint population, subsequently emerging as a population of peculiar interest. Therefore, these analyses, as far as extremely relevant, are not sufficiently powered to determine a statistical difference of efficacy between treatment regimens.

Moreover, very few EGFR-positive patients were previously treated with osimertinib (one in $\mathrm{ABCP}$, four in ACP, five in BCP) which is currently the standard of care of first-line and second-line therapy in EGFR-positive patients $(17,18)$. Therefore, these results cannot be translated to the current clinical practice. In addition, whereas the liver disease status is correctly stressed out, there is no mention of the brain involvement, that in NSCLC patients in general and in pretreated, EGFR-positive ones is of particular interest. Although no relevant safety issues emerged from the fourdrug arm, a careful evaluation of adverse events would be crucial in a real-world population of NSCLC, less selected than the present one (including approximately $45 \%$ of Eastern Cooperative Oncology Group (ECOG) performance status 0 patients) (10). Moreover, the approval of $\mathrm{ABCP}$ at national levels will likely require attentive financial estimations.

Nonetheless, the results of the IMpower 150 should be the starting point of future randomized trials on different treatment combinations specifically designed for these patients, which will provide additional insights on the best treatment after TKI failure. In this scenario, combination therapies involving ICI and anti-angiogenic agents could be extremely interesting as treatment strategy after thirdgeneration EGFR-TKI failure. Albeit ALK-positive cases were even less represented (13 patients treated in the ABCP arm) (9), the efficacy results of this treatment strategy still harbor potential clinical interest and are awaited.

In conclusion, the $\mathrm{ABCP}$ regimen might represent a potential therapeutic option for EGFR-mutated NSCLC patients after unsuccessful TKI therapy, patients with baseline liver metastases and those with low/negative PD-L1 expression, relevant patient subgroups. In the complex and "busy" scenario of the first-line treatment (including the postTKI one) of NSCLC, focusing on the specific subgroups of patients in which the synergistic effect of anti-angiogenesis and ICI added to chemotherapy provides meaningful improvements may be the key. Again, the global (clinical and financial) affordability of the four-agents regimen in routine practice will be a major point in determining its fate. Further randomised trials specifically designed for the precise reported patient populations are warranted, according to the latest preclinical evidence in the field of the interaction between tumor microenvironment and host immune system.

\section{Acknowledgments}

Funding: None.

\section{Footnote}

Provenance and Peer Review: This article is commissioned and reviewed by the Section Editor Wei Xu (Division of Respiratory Disease, Department of Geriatrics, the First Affiliated Hospital of Nanjing Medical University, Nanjing, China).

Conflicts of Interest: M Tiseo declares advisory boards and/ or speakers' fee for Astra-Zeneca, BMS, MSD, Boehringer Ingelheim, Takeda. The other authors have no conflicts of interest to declare.

Ethical Statement: The authors are accountable for all aspects of the work in ensuring that questions related to the accuracy or integrity of any part of the work are appropriately investigated and resolved.

Open Access Statement: This is an Open Access article distributed in accordance with the Creative Commons Attribution-NonCommercial-NoDerivs 4.0 International License (CC BY-NC-ND 4.0), which permits the noncommercial replication and distribution of the article with the strict proviso that no changes or edits are made and the original work is properly cited (including links to both the formal publication through the relevant DOI and the license). See: https://creativecommons.org/licenses/by-nc-nd/4.0/.

\section{References}

1. Reck M, Rodriguez-Abreu D, Robinson AG, et al. 
Pembrolizumab vs. chemotherapy for PD-L1-positive nonsmall-cell lung cancer. N Engl J Med 2016;375:1823-33.

2. Planchard D, Popat St, Kerr K, et al. Metastatic NonSmall-Cell Lung Cancer:ESMO Clinical Practice Guidelines for diagnosis, treatment and follow-up. Ann Oncol 2018;29:iv192-237.

3. Hellmann MD, Ciuleanu TE, Adam Pluzanski A, et al. Nivolumab plus Ipilimumab in Lung Cancer with a High Tumor Mutational Burden. N Engl J Med 2018;378:2093-104.

4. Gandhi L, Rodríguez-Abreu D, Gadgeel S, et al. Pembrolizumab plus chemotherapy in metastatic nonsmall-cell lung cancer. N Engl J Med 2018;378:2078-92.

5. Paz-Ares L, Luft A, Vicente D, et al. Pembrolizumab plus chemotherapy for squamous non - small-cell lung cancer. N Engl J Med 2018;379:2040-51.

6. Jotte RM, Cappuzzo F, Vynnychenko I, et al. Primary PFS and safety analysis of a randomized phase III study of atezolizumab + carboplatin + paclitaxel or nab-paclitaxel vs. carboplatin + nab-paclitaxel as $1 \mathrm{~L}$ therapy in advanced squamous NSCLC. J Clin Oncol abstr 2018;36:LBA9000.

7. Barlesi F, Nishio $M$, Cobo $M$, et al. Efficacy of atezolizumab (atezo) + carboplatin (carbo)/cisplatin (cis) + pemetrexed (pem) as 1L treatment in key subgroups with stage IV non-squamous non-small cell lung cancer (NSCLC). Ann Oncol 2018;29:mdy424.066.

8. Cappuzzo F, McCleod M, Hussein M, et al. Progression-free survival (PFS) and safety analysis from a randomised phase III study of carboplatin + nab-paclitaxel $(\mathrm{CnP})$ with or without atezolizumab (atezo) as first-line (1L) therapy in advanced non-squamous NSCLC. Ann Oncol 2018;29:mdy424.065.

9. Socinski MA, Jotte RM, Cappuzzo F, et al. Atezolizumab for first-line treatment of metastatic nonsquamous NSCLC. N Engl J Med 2018;378:2288-301.

10. Reck M, Mok TSK, Nishio M, et al. Atezolizumab plus bevacizumab and chemotherapy in non-small-cell lung cancer (IMpower150):key subgroup analyses of patients with EGFR mutations or baseline liver metastases in a randomised, openlabel phase 3 trial. Lancet Respir Med 2019;7:387-401

11. Perdrizet K, Leighl NB. The Role of Angiogenesis Inhibitors in the Era of Immune Checkpoint Inhibitors and Targeted Therapy in Metastatic Non-Small Cell Lung Cancer. Curr Treat Options Oncol 2019;20:21.

12. Manegold C, Dingemans AC, Gray JE, et al. The Potential of Combined Immunotherapy and Antiangiogenesis for the Synergistic Treatment of Advanced NSCLC. J Thorac Oncol 2017;12:194-207.

13. Cavanna L, Citterio C, Orlandi E, et al. Immune checkpoint inhibitors in EGFR-mutation positive TKI- treated patients with advanced non-small-cell lung cancer network meta-analysis. Oncotarget 2019;10:209-15.

14. Gainor JF, Shaw AT, Sequist LV, et al. EGFR Mutations and ALK Rearrangements Are Associated with Low Response Rates to PD-1 Pathway Blockade in Non-Small Cell Lung Cancer (NSCLC):A Retrospective Analysis. Clin Cancer Res 2016;22:4585-93.

15. Funazo T, Nomizo T, Kim YH. Liver metastasis is associated with poor progression-free survival in patients with non-small cell lung cancer treated with nivolumab. J Thorac Oncol 2017;12:e140-1.

16. Facchinetti F, Bordi P, Leonetti A, et al. Profile of atezolizumab in the treatment of metastatic non-small-cell lung cancer:patient selection and perspectives. Drug Des Devel Ther 2018;12:2857-73.

17. Soria JC, Ohe Y, Vansteenkiste J, et al. Osimertinib in Untreated EGFR-Mutated Advanced Non-Small-Cell Lung Cancer. N Engl J Med 2018;378:113-25.

18. Mok TS, Wu YL, Ahn MJ, et al. Osimertinib or PlatinumPemetrexed in EGFR T790M-Positive Lung Cancer. N Engl J Med 2017;376:629-40.

19. Meder L, Schuldt P, Thelen M, et al. Combined VEGF and PD-L1 Blockade Displays Synergistic Treatment Effects in an Autochthonous Mouse Model of Small Cell Lung Cancer. Cancer Res 2018;78:4270-81.

20. Koh YW, Lee SJ, Han JH, et al. PD-L1 protein expression in non-small-cell lung cancer and its relationship with the hypoxia-related signaling pathways:A study based on immunohistochemistry and RNA sequencing data. Lung Cancer 2019;129:41-7.

21. Tumeh PC, Hellmann MD, Hamid O, et al. Liver metastasis and treatment outcome with anti-PD-1 monoclonal antibody in patients with melanoma and NSCLC. Cancer Immunol Res 2017;5:417-24.

22. Hung MS, Chen IC, Lin PY, et al. Epidermal growth factor receptor mutation enhances expression of vascular endothelial growth factor in lung cancer. Oncol Lett 2016;12:4598-604.

23. Pacheco JM, Camidge DR. Searching for a chemoimmunotherapy signal in patients with non-smallcell lung cancer and EGFR mutations. Lancet Respir Med 2019;7:366-7.

Cite this article as: Rebuzzi SE, Facchinetti F, Tiseo M. Antiangiogenesis boosts chemo-immunotherapy in patients with EGFR mutations or baseline liver metastases: insights from IMpower150 study. Transl Cancer Res 2019;8(Suppl 6):S612S617. doi: $10.21037 /$ tcr.2019.06.21 